\title{
Valve sparing root replacement: reimplantation of the aortic valve
}

\author{
Gaby Aphram, Saadallah Tamer, Stefano Mastrobuoni, Gebrine El Khoury, Laurent de Kerchove \\ Department of Cardiothoracic and Vascular Surgery, Cliniques Universitaires Saint-Luc, Université Catholique De Louvain, Brussels, Belgium \\ Correspondence to: Laurent de Kerchove. Department of Cardiothoracic and Vascular Surgery, 10 Av. Hippocrate, 1200, Woluwe-Saint-Lambert, \\ Brussels, Belgium. Email: Laurent.dekerchove@uclouvain.be.
}

Submitted Jan 26, 2019. Accepted for publication Apr 05, 2019.

doi: 10.21037/acs.2019.04.05

View this article at: http://dx.doi.org/10.21037/acs.2019.04.05

\section{Clinical vignette}

The first patient in the video is an asymptomatic 65 -year-old male patient with permanent atrial fibrillation and permanent pacemaker for atrioventricular block of second degree. Transthoracic echocardiogram shows a tricuspid aortic valve (TAV) with eccentric aortic insufficiency (AI) grade 3+ towards the mitral valve indicating a right coronary leaflet prolapse. The left ventricular end diastolic (LVEDD) and end systolic (LVESD) diameter are 66 and $38 \mathrm{~mm}$, respectively. The left ventricular ejection fraction (LVEF) is $72 \%$. The ventriculoaortic junction (VAJ) diameter is $28 \mathrm{~mm}$, the sinus of Valsalva is $45 \mathrm{~mm}$, the sinotubular junction (STJ) is $40 \mathrm{~mm}$ and the ascending aorta is $42 \mathrm{~mm}$.

The second patient in the video is an asymptomatic 47-year-old male with no particular medical history. Transthoracic echocardiogram shows a bicuspid aortic valve (BAV) with right/left fusion, commissure orientation of $160^{\circ}$ and eccentric $\mathrm{AI}$ grade $3+$ towards the mitral valve indicating prolapse of the fused leaflet. The LVEDD and LVESD are 70 and $51 \mathrm{~mm}$, respectively. The LVEF is $54 \%$. The VAJ diameter is $30 \mathrm{~mm}$, the sinus of Valsalva is $45 \mathrm{~mm}$, the STJ is $36 \mathrm{~mm}$ and the ascending aorta is $40 \mathrm{~mm}$.

\section{Surgical technique}

Preparation and exposure were similar for both patients.

\section{Preparation}

The procedure is performed under general anesthesia, the patient is supine, arms along the body. A transesophageal echocardiographic (TEE) probe is placed. Surgery is performed through full sternotomy. Cardio-pulmonary bypass $(\mathrm{CPB})$ is performed through cannulation of the right atrium and distal ascending aorta. Cardiac arrest is obtained with intermittent normothermic antegrade blood cardioplegia.

\section{Exposure}

The ascending aorta is opened $1-2 \mathrm{~cm}$ above the STJ. Three traction stitches are placed at the tip of each commissure to assess valve coaptation and to expose the aortic root during external dissection. The size of the leaflets is assessed measuring their geometric height $(\mathrm{gH})$ and leaflet prolapse is confirmed comparing the free margins' effective height $(\mathrm{eH})$ using the Schäfers caliper.

\section{Surgical technique}

External aortic root dissection should reach the level of the basal ring except on the side of the right coronary sinus and the membranous septum where the dissection is limited by the right ventricular outflow tract and the roof of the right atrium, respectively. Once the non-coronary sinus is dissected, the sinus wall is resected leaving $5 \mathrm{~mm}$ of aortic rim along the cusp insertion. Right and left coronary buttons are isolated, leaving a generous patch of aortic wall around the ostia. The right and left ostial canula for cardioplegia are inserted and fixed in both ostia. The external root dissection is then continued from the left to the right sinus.

The size of the Gelweave Valsalva to be used is indicated by the height of the non-to-left commissure measured from the basal ring to the tip of the commissure (1). In the first patient of the video, a $28-\mathrm{mm}$ commissure height leaded us to use a $28-\mathrm{mm}$ Valsalva graft. Ten to $122-0$ 
Tycron sutures with pledget are then passed from inside out for the proximal suture line. Sutures are placed along the basal ring except at the area of the membranous septum where three of those stitches follow cusp insertion line and external root dissection to avoid damaging the bundle of His. The proximal suture line stitches are then passed along the caudal circumference of the neo-sinus portion of the Valsalva graft respecting the curvilinear contour of the proximal suture line. The graft is pulled down and the stitches are tied to ensure appropriate seating around the aortic annulus. The commissures are then reimplanted within the graft at $120^{\circ}$ each, at the highest level possible by applying more traction on the commissure than on the graft. In any case, the tip of each commissure must at least reach the neo-STJ of the Valsalva graft. Each 4-0 polypropylene suture, used to fix the commissures, is then used to perform the second suture line, a running suture starting from one commissure to the other to fix the remnant aortic sinus rim to the graft.

Right coronary leaflet prolapse is then corrected. To repair leaflet prolapse, the valve coaptation is re-assessed by applying traction on the commissures and by measuring eH with the caliper. All leaflets' free margins must reach an adequate $\mathrm{eH}$ of $9-10 \mathrm{~mm}$ and run parallel at the same level. Any leaflet with an eH below these values or that sits below the other(s) is considered as prolapsing and central plication is performed until the leaflet reaches an appropriate level.

The left coronary button is then reimplanted generally in the middle of the left neo-sinus of the graft using a running suture of 5-0 polypropylene. A strip of fresh autologous pericardium can be used on the coronary side to improve hemostasis. The right coronary button is then reimplanted with a similar technique. The right button is generally placed in the upper-left quadrant of the right neosinus, close to the right-to-non commissure. After coronary reimplantation, bulbar cardioplegia is given directly into the graft in order to distend the new aortic root allowing assessment of both hemostasis and valve competence by indirect signs such as root pressure and left ventricular dilatation. Finally, the graft is anastomosed to the ascending aorta which is eventually wrapped around its distal portion, using a Dacron strip, in order to avoid distal progression of dilatation. Distal wrapping is performed using a $2-\mathrm{cm}$ segment of the same graft which is passed around the distal aorta. The distal anastomosis is then performed including within the running suture the proximal end of the graft used for wrapping. After de-airing, the aorta is unclamped, and the distal end of the graft used for wrapping is pulled distally and fixed to the adventitial layer of the proximal aortic arch.

\section{Aortic repair in BAV}

The reimplantation technique in BAV generally follows the same sequence as in TAV, except for certain specificities that deserve to be highlighted. In the vast majority of BAV reimplantation, the commissures are placed at $180^{\circ}$ within the graft. This symmetrization of asymmetric BAV with raphe allows for the preservation of the mobility of the fused leaflet, which needs almost systematically to be plicated, in order to correct prolapse. Significant fibrous thickening of the raphe will be thinned to facilitate the plication and mobility of the fused leaflet.

\section{Repair assessment}

The quality of repair is then assessed by TEE, while the patient recovers physiological hemodynamic condition.

\section{Comments}

\section{Clinical results}

Between 1999 and 2017, a total of 440 consecutive patients underwent valve-sparing root replacement using the reimplantation technique (VSRR) in our institution (263 TAV, $177 \mathrm{BAV}$ ) (2). Cusp repair was performed in approximately $50 \%$ of TAV and $90 \%$ of BAV. Hospital mortality was $0.6 \%$ (3 patients including 2 with acute type A dissection). At 5 and 10 years, overall survival was $95.2 \%$ and $79.8 \%$, respectively. Freedom from valve-related death was $97.3 \%$ and freedom from aortic valve (AV) reoperation was $96.5 \%$ and $89.6 \%$, respectively, with no differences between TAV and BAV $(\mathrm{P}=0.1)$.

\section{Advantages}

Preservation of the native valve with valve-sparing procedure allows to reduce valve-related events, particularly thromboembolic and hemorrhagic complications related to mechanical valve and reoperation due to bioprosthesis degeneration, especially in young patients $(3,4)$. Moreover, survival, quality of life and AV hemodynamics seem to be better preserved with $\mathrm{AV}$ repair compared to replacement (3-5). 


\section{Caveats}

The technical complexity of valve sparing limits the diffusion of this procedure, especially in low-volume centers. Severe AV regurgitation, presence of calcifications or cusp retraction are all risk factors for early failure of the repair. Therefore, adequate patient selection and good mastery of the techniques are fundamental in ensuring long-term durability of the valve sparing.

\section{Acknowledgments}

None.

\section{Footnote}

Conflicts of Interest: The authors have no conflicts of interest to declare.

\section{References}

1. de Kerchove L, Boodhwani M, Glineur D, et al. A new simple and objective method for graft sizing in valvesparing root replacement using the reimplantation technique. Ann Thorac Surg 2011;92:749-51.

2. Mastrobuoni S, de Kerchove L, Navarra E, et al. Longterm experience with valve-sparing reimplantation technique for the treatment of aortic aneurysm and aortic regurgitation. J Thorac Cardiovasc Surg 2018. [Epub ahead of print].

3. de Meester C, Pasquet A, Gerber BL, et al. Valve repair improves the outcome of surgery for chronic severe aortic regurgitation: a propensity score analysis. J Thorac Cardiovasc Surg 2014;148:1913-20.

4. Ouzounian M, Rao V, Manlhiot C, et al. Valve-Sparing Root Replacement Compared With Composite Valve Graft Procedures in Patients With Aortic Root Dilation. J Am Coll Cardiol 2016;68:1838-47.

5. Aicher D, Holz A, Feldner S, et al. Quality of life after aortic valve surgery: replacement versus reconstruction. J Thorac Cardiovasc Surg 2011;142:e19-24.

Cite this article as: Aphram G, Tamer S, Mastrobuoni S, El Khoury G, de Kerchove L. Valve sparing root replacement: reimplantation of the aortic valve. Ann Cardiothorac Surg 2019;8(3):415-417. doi: 10.21037/acs.2019.04.05 